\section{Estudo da fauna de triatomíneos e da ocorrência de doença de Chagas em Monte Negro, Rondônia, Brasil}

\section{Study of the triatomine fauna and occurrence of Chagas disease in Monte Negro, Rondonia, Brazil}

Débora Cristina Massaro

Denise Silva Rezende

\section{Luis Marcelo Aranha Camargo}

Instituto de Ciências Biomédicas V Universidade de São Paulo (ICB5USP/MONTE NEGRO - RO)

Financimento: ICB5USP

Correspondência: Débora Cristina Massaro. Rua Braulino P. Gomes,1234. CEP: 78965-000. E-mail: deboramassaro@hotmail.com

\section{Resumo}

A doença de Chagas tem como agente etiológico o Trypanossoma cruzi, um protozoário flagelado que pode ser encontrado numa grande variedade de mamíferos e triatomíneos. O Estado de Rondônia, localizado na Amazônia Ocidental, possui um meio ambiente constantemente modificado pelas ações transformadoras do ser humano, resultando em um desequilíbrio, que pode facilitar a transmissão de inúmeros patógenos. Uma grande variedade e quantidade de palmáceas, em especial o babaçu, bem como mamíferos e triatomíneos, podem ser encontrados neste complexo ecossistema. Nesta pesquisa, a fauna de triatomíneos foi identificada em 225 babaçus e por meio de capturas peri e intradomiciliares. Foi realizado, concomitantemente, estudo de soroprevalência para doença de Chagas e a identificação da presença de T.cruzi no trato digestivo dos triatomíneos. Positividade ao T. cruzi foi verificada em $23,7 \%$ dos 652 triatomíneos coletados nos babaçus. Estes triatomíneos pertenciam ao gênero Rhodnius e foram classificados em 4 espécies: $R$. robustus, $R$. prolixus, $R$. pictipes e $R$. milesi. Nas capturas intradomiciliares, dez espécimes do Rhodnius robustus e uma de Panstrongylus geniculattus foram encontrados, sendo que $3 \%$ da população foi positiva para doença de Chagas. Na área pesquisada, há potencial de transmissão da doença de Chagas na forma endêmica devido a grande quantidade de triatomíneos, bem como alta freqüência de infecção destes triatomíneos, porém no momento deste estudo não se evidenciou a ocorrência da transmissão.

Palavras-chave: Trypanosoma cruzi. Epidemiologia. Amazônia. Doença de Chagas. Triatomíneos. 


\section{Abstract}

Trypanosoma cruzi is the etiologic agent of Chagas disease. It is a flagellate protozoan that can be found in a large variety of mammals and triatomines. The biodiversity of the state of Rondonia, located in theWestern Amazon, is constantly modified by man's transforming actions, which results in an unbalance that can facilitate the transmission of many pathogens. A great variety and quantity of native palms trees, especially the Babassu, as well as mammals and triatomines, can be found in the local ecosystem. In this survey, triatomine fauna was identified in 225 dissected Babassus, which were extra domiciled. In addition, peri-domiciled and intra-domiciled captures were done. Seroprevalence ocurrence of Chagas disease and identification of T. cruzi in the digestive tract of triatomines was performed. Positivity to T. cruzi was verified in $23.7 \%$ of the 652 triatomines collected from the Babassus. These triatomines belong to the Rhodnius genus and were classified into 4 species: $R$. robustus, $R$. prolixus, $R$. pictipes and $R$. milesi. In intra-domiciled captures, ten specimens of Rhodnius robustus and one of Panstrongilus geniculatus were found. Three per cent of the population was positive to Chagas disease. In the area surveyed, there is potential for endemic transmission of Chagas disease due to the large quantity of triatomines and to the high infection frequency of these triatomines. Nonetheless, this study did not provide evidence of the occurrence of endemic transmission at the present.

Keywords: Trypanosoma cruzi. Epidemiology. Rondonia. Triatomines. Chagas disease.

\section{Introdução}

A doença de Chagas ou tripanossomíase americana constitui uma das mais importantes endemias do Brasil e da América Latina $^{1}$, com o registro anual de 810.000 novos casos ${ }^{2}$. Com base nos dados do inquérito sorológico nacional, realizado entre 1975-1980, foi estimada em $4,2 \%$ a prevalência de chagásicos ${ }^{3,4}$.

$\mathrm{O}$ agente etiológico da doença de Chagas, Trypanosoma cruzi, é um protozoário flagelado da ordem Kinetoplastida e família Trypanosomatidae, que parasita mamíferos e tem como hospedeiros invertebrados numerosas espécies de hemípteros hematófagos da família Reduviidae ${ }^{5}$ e subfamília Triatominae. Todas as fases de seu ciclo evolutivo são hematófagas, vivem em média entre um a dois anos, com evolução de ovo, ninfa e adulto com grande capacidade de reprodução e, dependendo da espécie, com intensa resistência ao jejum ${ }^{1}$.

A transmissão da infecção ocorre principalmente pela deposição de fezes do vetor sobre os tecidos cutâneos e mucosas. Outras vias menos freqüentes são transfusões sanguíneas responsáveis por 5 a 20\% dos casos, via oral, via transplacentária, acidentes de laboratório, manipulação de animais infectantes e transplantes de órgãos ${ }^{6}$. A principal via de infecção pelo T. cruzi é a transmissão vetorial, correspondendo a $80 \%$ dos casos da doença de Chagas, ocorrendo em vastas regiões do México, América Central e América do Sul' ${ }^{2}$. A transmissão da doença de Chagas está relacionada à distribuição de vetores em uma determinada área, às características próprias dos vetores, assim como ao seu grau de antropofilia, números de parasitas eliminados com as fezes e urina, e ação desordenada do homem sobre o meio ambiente ${ }^{7}$.

A tripanossomíase na Região Amazônica e os riscos de sua transmissão têm sido motivos de preocupação ao longo dos tempos, dada a grande dispersão de vetores infectados e crescentes migrações humanas onde doença está relacionada com a degradação do ambiente natural e 
com o deslocamento de triatomíneos de seus ecótopos silvestres ${ }^{6,8,9}$.

O Estado de Rondônia, localizado a oeste da região Amazônica, abriga um ecossistema constantemente ameaçado pela ação transformadora do homem, resultando em um desequilíbrio que pode facilitar a aproximação do vetor ao homem e hipoteticamente facilitar a transmissão de vários patógenos, dentre estes a doença de Chagas ${ }^{9}$,

Trabalhos realizados já comprovaram a presença de focos naturais desta parasitose no Estado do Acre, na fronteira com o estado de Rondônia ${ }^{10,11}$. Este estudo afirma que, apesar de não ser endêmica, a Região Amazônica apresenta todos os elos da cadeia de transmissão da doença de Chagas ${ }^{12}$. Portanto, justifica-se a averiguação da ocorrência de transmissão da doença de Chagas em Rondônia.

\section{Objetivos}

O objetivo foi identificar a fauna de triatomíneos encontrados no extradomícílio (babaçus), peri-domicílio e intradomicilio; verificar a ocorrência de infecção por Trypanosoma cruzi no trato digestivo do triatomíneo, realizar estudo de soro prevalência da doença de Chagas na população estudada e criar subsídios para eventual controle da endemia na região.

\section{Materiais e Métodos}

O estudo foi realizado no município de Monte Negro (S 10 15’35”, W 63 18'06”), situado no Estado de Rondônia, com uma população estimada de 16.874 habitantes (sendo 60\% em área rural), ocupando uma área de $1.931 \mathrm{~km} 2$ (densidade populacional de $8,7 \mathrm{hab} / \mathrm{km} 2$ ) e distando $250 \mathrm{~km}$ da capital Porto Velho ${ }^{21}$.

Tem como média anual de precipitação pluviométrica 1800 a $2200 \mathrm{~mm}$, maior que a do Estado, e temperatura média de 25,8 C, próxima à do Estado, oscilando entre 12 e $37^{\circ} \mathrm{C}$, e umidade relativa entre 70 a $80 \%$ durante o $\mathrm{ano}^{22}$.
O município de Monte Negro é composto por 15 linhas (estradas vicinais que cortam a BR 421 e outras linhas menores denominadas "travessão", que surgem de cortes em linhas de maior porte), como mostram os Mapas 1 e 2. No trabalho realizado foram amostrados 15 sítios da área rural, aleatoriamente (Mapa 3), localizados em diferentes linhas. As famílias residentes nessas propriedades foram cadastradas.

\section{Coleta extradomiciliar}

Em cada propriedade foram dissecados 15 babaçus “(Orgygnia speciosa) (permissão IBAMA/2002) para captura de triatomíneos. As brácteas foram retiradas uma por vez na busca de triatomíneos.

\section{Coleta peridomiciliar}

O peridomícilio (galinheiro, pocilgas, tulhas, etc) foi vistoriado através de busca ativa de triatomíneos.

\section{Coleta intradomiciliar ou busca ativa}

Em todas propriedades foram distribuídos mostruários com espécimes de triatomíneos e material de coleta aos moradores para que pudessem conhecer alguns espécimes e eventualmente capturá-las se as encontrassem no intradomicilio e/ou peridomícilio.

Os triatomíneos foram identificados através de caracteres morfológicos externos, utilizando-se como ferramenta a chave dicotômica elaborada por Lent \& Wygodzinsky, 1979. Para a detecção de T. cruzi em tubo digestivo, os triatomíneos tiveram seu tubo digestivo dissecado e examinado para se verificar a positividade quanto à presença de tripanossomas. As amostras positivas foram semeadas em tubos contendo meio de cultura bifásico BAB sangue+LIT. Foram preparados esfregaços em lâminas de tubo digestivo de triatomíneos e amostras de culturas em fase logarítmica e estacionária, para a identificação de T. cruzi através da caracterização morfológica. Os esfregaços foram 


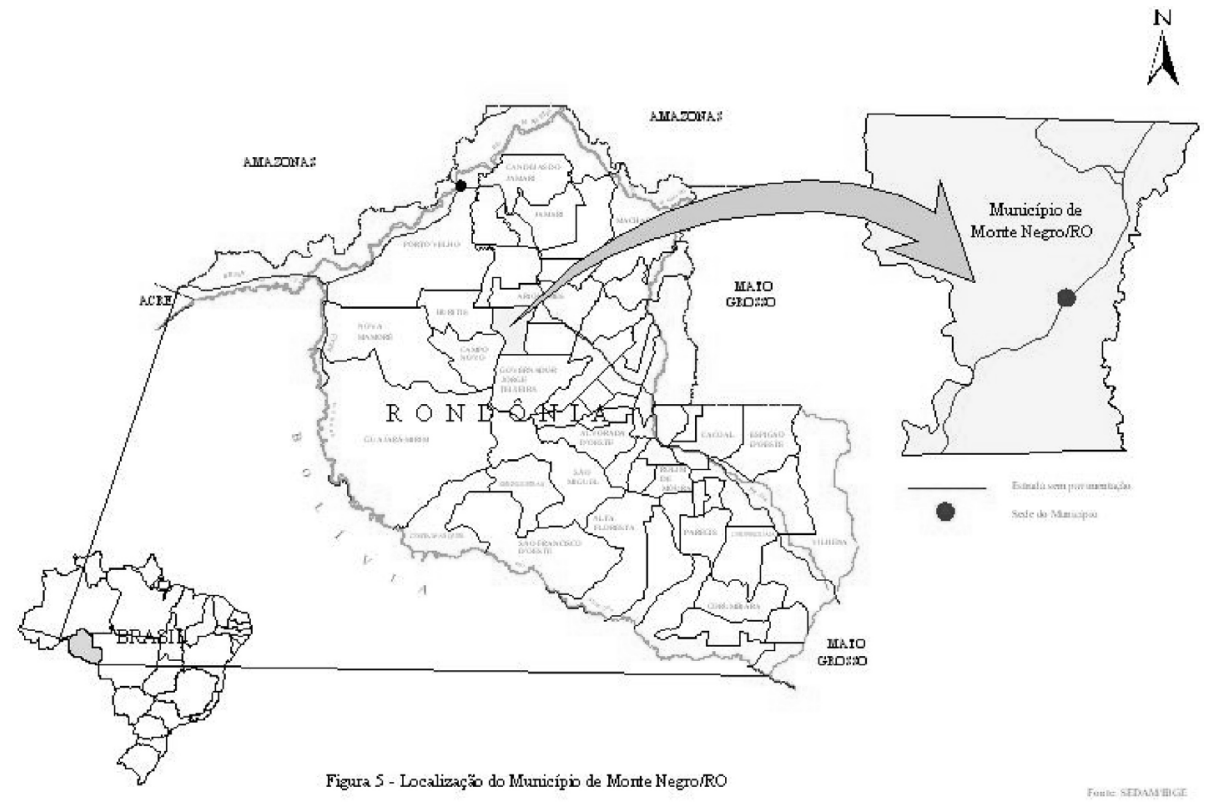

Mapa 1 - Localização do município de Monte Negro/ RO

Map 1 - Location of the municipality of Monte Negro/RO

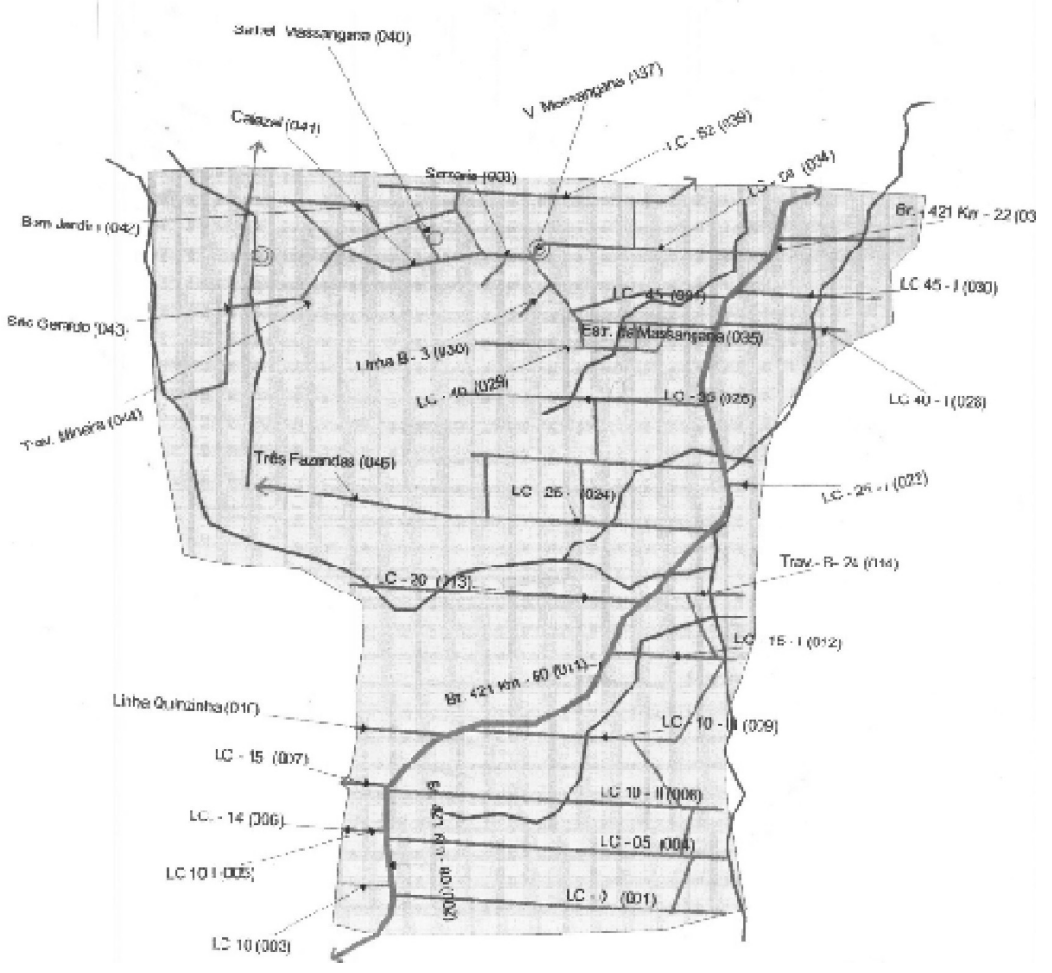

Fonte: FUNASA

Mapa 2 - Localização das linhas do município de Monte Negro, Rondônia.

Map 2 - Location of the roads of the municipality of Monte Negro, Rondonia. 


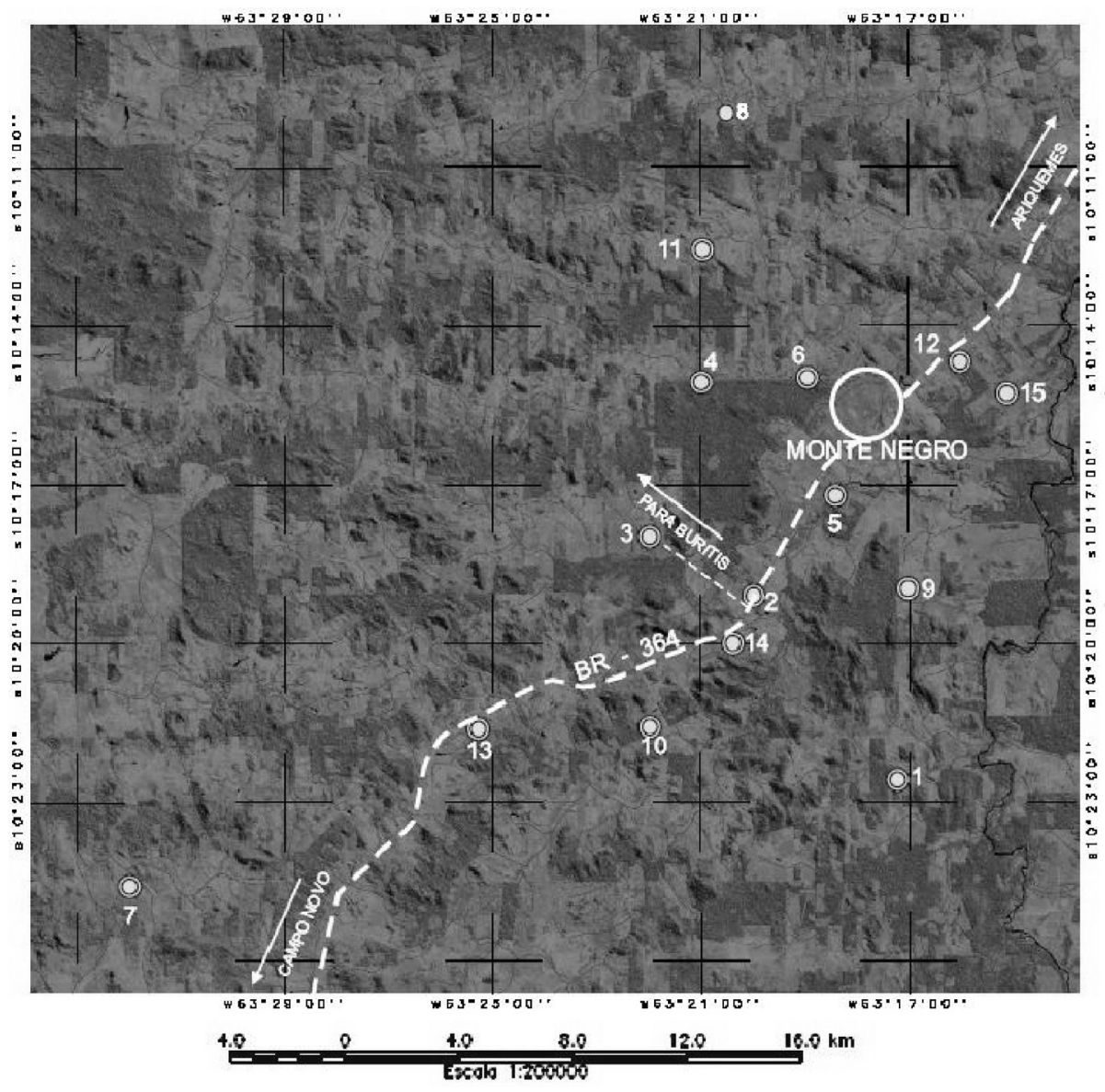

Mapa 3 - Foto de satélite mostrando as propriedades Monte Negro / RO.

Map 3 - Satellite picture showing the properties of Monte Negro/RO.

fixados com metanol e corados com Giemsa e observados em microscópio de luz .

\section{Abordagem dos seres humanos}

Os pacientes residentes nas propriedades em estudos passaram por exames médicos e laboratoriais voluntariamente, visando identificar os casos crônicos de doença de Chagas, assintomáticos ou não. Na ocasião, assinaram termo de consentimento esclarecido, sendo preenchida uma ficha clínico-epidemiológica por profissional médico. Foram feitas coletas de sangue, colhidos $10 \mathrm{ml}$ de sangue por venopunção e centrifugados por 10 minutos a $2500 \mathrm{RPM}$, e aliquotados $3 \mathrm{ml}$ de soro para análise sorológica para a detecção de anticorpos anti $T$. cruzi pela técnica de imuno ensaio enzimático (ELISA) e teste confirmatório por imunofluorescencia indireta (IgG).

\section{Clearance Ético}

O presente estudo foi submetido ao comitê de ética do Centro de Pesquisas em Medicina Tropical de Rondônia.

Aprovado pelo Comitê de Ética em Pesquisa do Centro de Pesquisa em Medicina Tropical, Secretaria do Estado da Saúde, Governo do Estado de Rondônia, Ofício 009/2005

\section{Resultados}

No estudo sobre a fauna de triatomíneos e a ocorrência de doença de Chagas, no município de Monte Negro, Rondônia, no 
período de março de 2003 a março de 2004, foram visitadas 15 propriedades, dissecados 225 babaçus e realizados 99 exames clínicos epidemiológico e laboratoriais nos habitantes desta área.

\section{Perfil das propriedades e habitações}

O tempo de colonização das propriedades rurais apresenta média e mediana de 24 anos e desvio padrão de 3,6 anos. Distam de 3,5 a 37 $\mathrm{km}$ do núcleo urbano, com média e mediana de 12,2 km e desvio padrão de 9 km. Ocupam em torno de 50 a 130 habitantes, os quais desenvolvem atividades agropecuárias.

Os proprietários rurais têm renda mensal variando entre 2 e 8 salários mínimos e possuem moradias com padrão uniforme, caracterizadas por estrutura de madeira, cobertura de telha de amianto ou telhas de barro (Figura 1) e no peridomicilio foi observado a presença de benfeitorias (galinheiro, pocilga, estábulo e tulha), 95\% delas cobertas com folhas de babaçu.

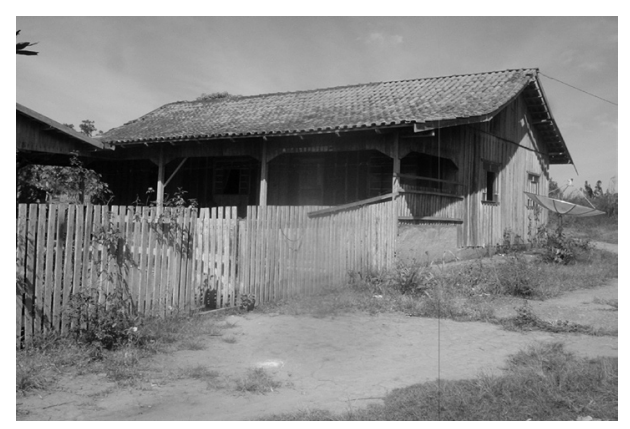

Figura 1 - Residência típica encontrada nas propriedades rurais, Monte Negro, Rondônia, 2003.

Figure 1 - Example of houses found in the rural areas of Monte Negro, Rondonia, 2003

Salienta-se que as residências localizamse em meio a pastagens margeadas por fragmentos pouco preservados de floresta.

\section{Perfil da população}

A população estudada é composta na maioria por imigrantes oriundos de várias regiões. Apenas $46 \%$ da população abordada é natural de Rondônia e a maior parcela desta população é do sexo masculino.

A média de idade da população é de 32,1 anos e o tempo médio de residência no Estado de Rondônia é de 19,1 anos, sendo de 12,7 anos no município de Monte Negro.

\section{Inquérito sobre a fauna em babaçus}

Na dissecação dos 225 babaçus foi observada e anotada em planilha uma grande diversidade de espécies nativas e turistas de animais, classificadas em 3 filos, 9 classes e 36 ordens.

\section{Coleta extradomiciliar em babaçus}

Os babaçus dissecados localizavam-se em média a uma distância de 10 a 800 metros do domicílio (Tabela 1) e de 50 a 1000 metros da floresta, $85 \%$ dos babaçus foram encontrados positivos para triatomíneos totalizando 652 espécimes coletados (ninfas e adultos) no período de estudo. A maior parte dos babaçus dissecados foi encontrada com ninhos de pássaros e mamíferos (Tabela 2).

Os triatomíneos foram coletados no terço superior das palmáceas, nas axilas das folhas, nas brácteas que envolvem os pendões. Foram encontrados em média 51 triatomíneos por propriedade e 3,4 por babaçu. As propriedades tinham características semelhantes, tais como domicílios em meio a pastagens, cercadas por babaçus, porém diferiam em relação à distância entre a residência, o babaçu e a floresta.

Como foi observado na Tabela 1, os recursos alimentares disponíveis nas proximidades da residências ( palmáceas) estão diretamente relacionados com a ocorrência de triatomíneos, havendo uma correlação inversamente proporcional entre a distância da residência e o número médio de barbeiros por babaçu (teste de Kruskai-Wallis com $\mathrm{p}<0,00001)$. Na Tabela 2 verificamos que há um maior número médio de barbeiros em babaçus onde foram encontrados mamíferos do que nos babaçus que apresentavam somente pássaros, havendo uma maior média de barbeiros por babaçu 
Tabela 1 - Distribuição do número médio de triatomíneos coletados em babaçus nas propriedades amostradas em Monte Negro, Rondônia, 2003.

Table 1 - Distribution of the average number of triatomines collected in Babassus in the properties sampled in Monte Negro, Rondonia, 2003.

\begin{tabular}{|c|c|c|c|}
\hline DRB * & $\mathrm{TT}^{* *}$ & $\mathrm{TBE}^{* * *}$ & MTEB ${ }^{* * * *}$ \\
\hline $0-110$ & 293 & 53 & 5,5 \\
\hline $110-211$ & 223 & 79 & 2,8 \\
\hline $211-310$ & 51 & 21 & 2,4 \\
\hline $310-411$ & 49 & 30 & 1,6 \\
\hline $411-510$ & 22 & 15 & 1,4 \\
\hline $610-711$ & 10 & 15 & 0,6 \\
\hline$>711$ & 4 & 12 & 0,3 \\
\hline
\end{tabular}

Tabela 2 - Distribuição do número de triatomíneos coletados em babaçus relacionando com a ocorrência de ninhos de pássaros e mamíferos nas propriedades amostradas de Monte Negro, Rondônia, 2003.

Table 2 - Distribution of the number of triatomines collected in Babassus related to the occurrence of bird nests and mammals in the properties sampled in Monte Negro, Rondônia, 2003.

\begin{tabular}{|lccc|}
\hline & TT $^{*}$ & TBE & MTEB *** \\
\hline Pássaros e mamíferos & $456(70 \%)$ & $120(53,4 \%)$ & 3,8 \\
Mamíferos & $110(17 \%)$ & $49(21,8 \%)$ & 2,2 \\
Pássaros & $65(10 \%)$ & $22(9,8 \%)$ & 2,9 \\
Sem pássaros e mamíferos & $19(3 \%)$ & $34(15 \%)$ & 0,6 \\
\hline
\end{tabular}

*TT = Total de triatomíneos / total of triatomines

** TBE $=$ Total de babaçus examinados / total of Babassus examined

*** MTEB = Média de triatomíneos encontrados por babaçu / Average of triatomines found per Babassu

em relação à presença de qualquer um deles (aves ou mamíferos), em comparação com a ausência de pássaros e mamíferos (teste de Kruskal-Wallis com $\mathrm{p}<0,00001$ ). Os triatomíneos coletados pertencem ao gênero Rhodnius, classificados em 4 espécies: Rhodnius robustus (83,1\%), Rhodnius prolixus (10,8\%), Rhodnius pictipes $(4,6 \%)$, Rhodnius milesi (1,5\%).

\section{Coleta no peri-domicílio}

Em cada propriedade foi vistoriado o peridomícilio (pocilgas, galinheiros e tulhas), não sendo encontrados espécimes de triatomíneos.

\section{Coleta intradomiciliar ou busca ativa}

Após a entrega de mostruários contendo espécimes de triatomíneos, os proprietários foram orientados a capturá-los. Durante o período da pesquisa, em quatro das 15 propriedades foram encontradas 11 espécimes. Destas, dez espécimes foram Rhodnius robustus e um Panstrongylus geniculatus, todas as espécimes adultas, não sendo detectadas formações de colônias.

Na propriedade do produtor JSP, que dista $12 \mathrm{Km}$ do centro urbano, com uma área total de 112 ha, foram coletados 7 triatomíneos no intradomicílio, todos no período noturno. Os triatomíneos foram encontrados em vários locais da casa, inclusive sobre o mosquiteiro. A propriedade apresenta as seguintes características: topografia do terreno em declive acentuado, cercado de morros, estando a residência localizada na parte mais baixa do terreno, em meio à pastagem. O domicílio é de madeira, coberto de 
amianto e com um piso a uma altura de 1,5 metros do chão, feito de madeira, sendo este local um abrigo para animais domésticos (cães, gatos e aves).

Esta residência apresenta as benfeitorias semelhantes às demais propriedades, tais como pocilga, galinheiro, tulha e curral, todos cobertos por folhas de palmáceas e localizados a uma distância média de aproximadamente 7 metros da residência.

Na propriedade que faz divisa com o produtor JSP foram encontrados na varanda da residência dois insetos: um Rhodnius robustus e um Panstrongylus geniculatus. Apesar de a propriedade não fazer parte da amostragem abordada na pesquisa, achou-se pertinente a inclusão da informação. O proprietário identificou os espécimes de triatomíneos através do mostruário do vizinho. A topografia do terreno também é de declive acentuado e a residência se localiza na parte baixa.

Na propriedade de número 15 , do produtor GCO, que dista $12 \mathrm{~km}$ da área urbana, com área total de 80 hectares de relevo plano, foi capturado um espécime de Rhodnius robustus atrás de um armário da cozinha da casa.

Outro triatomíneo foi encontrado no intradomicílio do proprietário AAF, que dista $3,5 \mathrm{~km}$ da área urbana.

\section{Infecção dos triatomíneos por T. cruzi}

Dados resultantes da microscopia óptica realizada junto ao Instituto de Ciências Biomédicas II - Departamento de Parasitologia da Universidade de São Paulo (Tabela 3) totalizaram $23,7 \%$ de triatomíneos positivos para T. cruzi.

\section{Estudo de soroprevalência}

Através de questionários foram coletadas informações dos indivíduos, com objetivo de avaliar as condições clinicas e epidemiológicas da população amostrada. Os moradores das propriedades estudadas foram submetidos a sorologia para anticorpos anti - T. cruzi através do método imunoensaio enzimático (ELISA) e
Tabela 3 - Índice de positividade de triatomíneos para T. cruzi, por propriedade, de março a novembro de 2003. Monte Negro, Rondônia, 2003.

Table 3 - Triatomine positivity to T. cruzi, per property, from March to November 2003. Monte Negro, Rondonia, 2003.

\begin{tabular}{lccc}
\hline PROP & $\mathrm{TI}^{* *}$ & $\mathrm{TC}^{* * *}$ & $\%$ \\
\hline 1 & 12 & 18 & 66,7 \\
2 & 1 & 4 & 25 \\
3 & 0 & 1 & 0 \\
4 & 0 & 6 & 0 \\
5 & 3 & 5 & 60 \\
6 & 4 & 14 & 28,6 \\
7 & 0 & 7 & 0 \\
8 & 2 & 8 & 25 \\
9 & 6 & 18 & 33,3 \\
10 & 16 & 17 & 94,1 \\
11 & 0 & 20 & 0 \\
12 & 11 & 44 & 25 \\
13 & 2 & 16 & 12,5 \\
14 & 1 & 31 & 3,2 \\
15 & 1 & 40 & 2,5 \\
Total & 59 & 249 & 23,7 \\
\hline PROP* Propra
\end{tabular}

PROP * $=$ Propriedade estate

$\mathrm{TI}^{* *}=$ Triatomíneos infectados / infected triatomine TC ${ }^{* * *}=$ Triatomíneos coletados / collected triatomine $\%{ }^{* * *}=$ Percentagem de triatomíneos infectados por propriedade / percentage of infected triatomine per estate

do método de imunofluorescência indireta (IgG). Foram examinados 99 indivíduos, e destes $70,7 \%$ não relatam antecedentes chagásicos. No total, $3 \%$ apresentaramse positivos para doença de Chagas. Os indivíduos positivos foram convocados para uma consulta médica para a leitura dos resultados.

O primeiro paciente soro-reagente (ELISA e imunofluorescência indireta) é do sexo masculino, imigrante da região noroeste do Paraná, com 54 anos de idade, e residindo em Rondônia há 27 anos, sendo 23 na mesma propriedade. O mesmo não apresenta sintomas nem antecedentes chagásicos, e foi diagnosticado através deste estudo.

$\mathrm{O}$ segundo paciente soro reagente é do sexo feminino, imigrante do Estado de Minas Gerais, esposa do primeiro paciente e tem 53 anos. Reside em Rondônia há 27 anos e há 23 reside nesta propriedade. Submeteuse a uma transfusão de sangue há 32 anos e 
não possui antecedente chagásico. Também não apresenta sintomas e foi diagnosticada através deste estudo.

O terceiro paciente, imigrante do Estado do Pará, sexo masculino, tem 52 anos, residente há 19 anos no Estado de Rondônia e há 11 anos nesta propriedade. Refere que, ao doar sangue em 1977, constatou a doença de Chagas. O paciente foi submetido ao exame eletrocardiográfico convencional e dinâmico (Holter de 24 horas), sendo constatada arritmia cardíaca.

\section{Discussão}

O perfil das propriedades e habitações abordadas no estudo são atípicas, em comparação com as residências descritas em outras regiões endêmicas. O padrão habitacional contrasta com o trabalho de alguns autores, que relatam que a população sob o risco da transmissão da doença de Chagas sobrevive em estado precário, com residências construídas de pau-a-pique, cobertas de palha e rodeadas de palmáceas, resultado da baixa condição econômica e social da comunidade ${ }^{10,14}$.

As residências amostradas no estudo encontram-se inseridas em meio a pastagens, rodeadas por floresta e com abundância de babaçus, sendo as folhas desta palmeira abrigo de triatomíneos, e utilizam as palhas como cobertura de benfeitorias e criação de animais domésticos no peridomícilio.

Estudo realizado na região de Bambuí, Minas Gerais, observou que o processo de colonização desordenada provocou bruscas mudanças na vegetação e rareamento dos animais silvestres. Isso fez com que novos ciclos de transmissão entre o homem e os animais domésticos passassem a fazer parte da cadeia epidemiológica da doença de Chagas, com possibilidade de intercambio do $T$. cruzi entre os ciclos silvestre e doméstico ${ }^{15}$. O Estado de Rondônia em comparação ao descrito acima, teve sua ocupação de forma semelhante no início na década de 70, com intenso desmatamento para introdução da atividade agropecuária, causando bruscas mudanças na vegetação e impacto na fauna silvestre, podendo acarretar o ciclo de transmissão do T. cruzi com a aproximação dos triatomíneos e animais silvestres ao domicílio, fato que não foi constatado no presente estudo, devido à ausência de colônias de triatomíneos no intradomicílio.

\section{Inquérito sobre a fauna de babaçu}

Foram observadas e coletadas uma grande diversidade de animais no babaçu, incluindo mamíferos, potenciais reservatórios para Trypanosoma cruzi, e espécimes de triatomíneos. Vários estudos têm salientado a importância de palmáceas como ecótopos naturais destes vetores, principalmente da espécie do gênero Rhodnius, importantes na transmissão da doença de Chagas ${ }^{16,17}$. Neste estudo, todos os triatomíneos coletados em palmeiras pertenciam ao gênero Rhodnius, confirmando resultados de outros trabalhos realizados em diferentes áreas do Brasil que têm mostrado altas taxas de infestação de Rhodnius em palmáceas ${ }^{9,18}$.

\section{Inquérito sobre Triatomíneos}

No Brasil existem pelo menos 44 espécies de triatomíneos identificados, sendo a maioria silvestres ${ }^{6}$. Na Amazônia foram encontradas 18 espécies de triatomíneos, 10 infectadas com T. cruz ${ }^{19}$. Em Rondônia, na região de Monte Negro, encontramos o gênero Rhodnius, com altas taxas de infecção por T. cruzi, $23,7 \%$, representando um potencial vetor para tripanossomíase na região.

\section{Coletas extradomiciliar}

O babaçu, planta originária da região amazônica e da mata Atlântica da Bahia, é encontrado em grande quantidade em Rondônia. Das palmáceas dissecadas foram encontradas $85 \%$ infestadas por triatomíneos. Por ser uma planta hospedeira, representa uma combinação de abrigo, recurso alimentar, desenvolvimento e procriação de triatomíneos (micro-habitat) ${ }^{16}$.

O gênero Rhodnius representa $100 \%$ dos triatomíneos coletados em palmáceas no es- 
tudo, o que está de acordo com os resultados descritos em outras regiões do Brasil ${ }^{20}$.

Nas propriedades onde os babaçus se localizam mais próximos da residência, com distancia média variando de 10 a 210 metros, foram coletados $79 \%$ de triatomíneos. Este número é relativamente alto se comparado com o número de triatomíneos encontrados em babaçus com distâncias de 211 a mais de 700 metros, onde foram coletados $21 \%$ de triatomíneos.

Estefato pode ser explicado pelaintensificação da ação antrópica, provocando mudanças nos ecótopos naturais, tanto de mamíferos silvestres quanto de triatomíneos, importantes elos da cadeia de transmissão do T. cruzi. O desequilíbrio, conseqüentemente, propicia a aproximação dos animais silvestres e dos triatomíneos ao peridomícilio em busca de alimento, os triatomíneos encontram disponibilidade de alimento e têm os babaçus como abrigo ${ }^{15}$. As palmeiras são importantes ecótopos naturais de triatomíneos, sendo consideradas indicadores ecológicos da presença de triatomíneose hospedeiros dos ciclos silvestres de transmissão do T.cruzi ${ }^{16,21}$.

Um fator que determina o tamanho das colônias de triatomíneos é a disposição de alimentos encontrados. Foi observado que havendo a presença de nidificação e mamíferos nos babaçus a infestação de triatomíneos foi significante $(3,8 \%)$, esta ocorrência não é aleatória, é fortemente influenciada pela presença de ninhos de aves e mamíferos, pois são fontes diretas de alimentos para os insetos ${ }^{17}$.

Foram encontradas neste trabalho as espécies $R$. robustus, $R$. pictipes, $R$. prolixus e $R$. milesi associados ao babaçu. O mesmo foi observado em uma espécie diferente de palmácea (Attalea maripa), conhecida como importante ecótopo para as espécies $R$. pictipes e $R$. prolixus em regiões de vegetação desmatada de Manaus ${ }^{11}$.

Neste trabalho a espécie $R$. robustus foi a mais prevalente nas coletas extradomiciliares (babaçu) com 83,1\% . No ambiente silvestre $R$. robustus está associado às palmeiras e já foram encontrada em peridomícilios e domicílio infectado pelo
T. cruzi considerado um vetor potencial na transmissão silvestre ${ }^{22}$.

Rhodnius prolixus foi a segunda espécie mais prevalente, representando $10,8 \%$ dos barbeiros coletados em palmáceas em áreas de pastagens onde foi observada a presença de mamíferos (marsupiais e ratos), aves e répteis. A domiciliação do $R$. prolixus foi conseqüência do desmatamento, tornandose um importante vetor para a doença de Chagas na América Central, onde foi relatada a transmissão domiciliar ${ }^{23}$.

A espécie $R$. pictipes foi a menos prevalente em palmáceas, representando 4,6\%. A espécie é arborícola, encontrada principalmente em palmeiras, pois está associada a refúgios de morcegos. É uma espécie silvestre de ampla distribuição na América do Sul. No Brasil tem sido encontrada no Estado do Amazonas ${ }^{24}$.

\section{Coletas intradomiciliares}

Nas coletas intradomiciliares, Rhodnius também foi o gênero mais predominantemente encontrado, invadindo o intradomicílio e representando $91 \%$ dos espécimes coletados. $R$. robustus é um dos potenciais vetores dentro da transmissão silvestre do T. cruzi, por ser encontrado no intradomicilio $^{21}$.

Panstrongylus geniculatus foi coletado invadindo o intradomicilio. Esse gênero é preferencialmente associado a tocas de roedores $^{50,51}$. Os vôos foram mais observados no período seco ou no início do período chuvoso $^{11}$, e no presente trabalho foram nesta época encontrados no intradomicilio.

Neste estudo não foi detectada colônia de triatomíneos nas casas, e por isso ainda não está havendo domiciliação. As espécies encontradas pelos proprietários invadindo as residências são adultas, e portanto com adaptação para o vôo, provenientes do ambiente silvestre ${ }^{11}$.

\section{Inquérito humano}

Em nossos estudos, 3\% dos pacientes apresentaram-se soros reagentes para $T$. 
cruzi. Estes pacientes são oriundos de áreas endêmicas e residem em Rondônia há mais de 20 anos, apresentando idade média superior aos 50 anos. Não foi detectado soro reagente em crianças e jovens, sugerindo que não está havendo transmissão vetorial na região. Um quadro semelhante foi retratado em Roraima onde indivíduos adultos migrantes tiveram resultados sorológicos positivos para Chagas, apesar do resultado, a área não é considerada endêmica ${ }^{12}$. Contrastando com o município de Rio Negro, estado do Amazonas, em que dois pacientes autóctones foram a óbito por miocardiopatia chagásica crônica, estas pessoas nasceram e viveram toda vida na região tendo sido picadas numerosas vezes por triatomíneos silvestres na área ${ }^{25}$.

Apesar de não constatar a ocorrência de casos autóctones na região de estudo, observamos a presença de todos os elos na cadeia de transmissão da doença de Chagas. E, com a crescente antropização, o homem pode acabar interferindo no ciclo enzoótico da doença, expondo-se ao risco e provavelmente incrementando a casuística da doença humana nesta região. Portanto, faz-se necessário um programa de vigilância epidemiológica para evitar a domiciliação de triatomíneos nesta região e o risco de transmissão vetorial.

\section{Conclusão}

Este trabalho constitui um estudo de caráter preliminar, objetivando descrever a epidemiologia da doença de Chagas na região estudada.

O presente estudo permite concluir que:

- Na fauna identificada de triatomíneos do Município de Monte Negro/RO foram capturados dois gêneros de triatomíneos: Rhodnius e Panstrongylus. Nas buscas ativas extradomiciliares foram classificados em 4 espécies: Rhodnius robustus (83,1\%), Rhodnius prolixus (10,8\%), Rhodnius pictipes (4,6\%), Rhodnius milesi (1,5\%) Nas buscas intradomiciliares foram capturados
Panstrongylus geniculatus e Rhodnius robustus.

- Há um alto índice de triatomíneos no extradomicilio, com $85 \%$ dos babaçus infestados, onde $23,7 \%$ estão contaminados positivamente com T cruzi.

- Espécimes intradomiciliares não são freqüentes, sendo responsáveis por $1,7 \%$ das capturas dos insetos adultos, não havendo colônias.

- Não foram encontrados triatomíneos no peridomícilio.

- Três por cento dos pacientes estudados foram positivos para doença de Chagas, porém são oriundos de áreas endêmicas. Apenas um paciente possui antecedente chagásico, e não se pode comprovar que a parasitose esteja sendo transmitida em Monte Negro, RO. A ausência de casos nas faixas etárias mais jovens reforça a hipótese de que a infecção não foi adquirida em Monte Negro.

- A região pode se tornar potencial de transmissão da doença de Chagas na forma endêmica, devido à abundância de triatomíneos e à alta freqüência com que se encontram infectados.

- Recomenda-seumestudoepidemiológico e entomológico de maior abrangência em diferentes regiões do Estado para se investigar este fenômeno.

- Sugere-se a investigação das cepas circulantes na região.

- As palmeiras da espécie Orbygnia speciosa representam um indicador em potencial da presença de triatomíneos.

- Quanto mais próximos os babaçus do domicilio, maior o número de triatomíneos capturados.

Portanto, faz-se necessária a realização de vigilância epidemiológica na população e implantação de programas de controle epidemiológico para evitar a transmissão da doença de Chagas. Tendo por base o consenso brasileiro em doença de Chagas (2005), sugere-se a aplicação de alguns procedimentos:

Vigilância entomológica por parte dos moradores para que na ocorrência de colonização ou eventual invasão o sistema de 
saúde seja acionado, a detecção de casos febris através de exames hemoscópicos realizados nos postos de saúde para detecção da malária e a melhoria das estruturas físicas no peridomicílio para evitar a aproximação e colonização dos vetores.

\section{Referências}

1. Jurberg J, Galvão C, Noireau F, Carcavalho RU, Rocha DS, Lent H. Uma Iconografia dos Triatomíneos (Hemíptera: Reduviidae). Entomol Vect 2004; 11(3): 454-94, disponível em www.ugf.br [acessado em 2003].

2. Aaras R, Gomes I, Veiga M, Melo A. Transmissão vetorial da doença de Chagas em Mulungu do Morro, Nordeste do Brasil. Rev Soc Bras Med Trop 2003; 36: 359-63.

3. Secretaria de Vigilancia em Saúde do Ministerio da Saúde. Consenso Brasileiro em doença de Chagas. Rev Soc Bras Med Trop 2005; 38(3): 1-29.

4. Camargo ME, Silva GR, Castilho EA, Silveira AC. Inquérito sorológico da prevalência da infecção chagásica, no Brasil, 1975/1980. Rev Inst Med Trop 1984; 26: 192-204.

5. Chagas C. Nova Tripanozomiaze humana. "Estudo sobre a morfologia e o ciclo evolutivo do Schizotrypanum cruzi, agente etiológico de nova entidade mórbida do homem”. Mem Inst Oswaldo Cruz 1909: 1 (S1): 159-218.

6. Coura JR. Tripanosomose, Doença de chagas, Cienc Cult 2003; 55: 1 .

7. Silveria AC, Feitosa VR, Borges R. Distribuição de triatomíneos capturados no ambiente domiciliar, no periodo 1975/83, Brasil. Rev Bras Malariol D Trop 1984; 36: $312-5$

8. Dias JCP, Prata A, Schofield CJ. Doença de Chagas na Amazonia: esboço da situação atual e perspectiva de prevenção. Rev Soc Bras Med Trop 2002; 35(6): 669-78.

9. Teixeira ARL, Monteiro OS, Rebelo, JM, Arganarz ER, Vieira, D, Pires LL et al. Emerging Chagas Disease: Trophic Network and Cycle of Transmission of Trypanosoma cruzi from Palm trees in the Amazon, disponivel em http://www.medcape.com/ viewarticle/414367,2001 [acessado em 2003].

10. Barata JMS, Rocha RM, Rodrigues VL. Primeiro caso autóctone de tripanossomíase no Estado do Acre (Brasil) e sua correlação com as cepas isoladas do caso humano e de triatomíneos silvestres da área. Rev Saúde Pública 1988; 22: 390-400.

11. Naiff MF, Naiff RD, Barrett TV. Vetores selváticos de doença de Chagas na área urbana de Manaus (AM): atividade de vôo nas estações secas e chuvosas. Rev Soc Bras Med Trop 1998; 31: 103-5.

12. Luitgards-Moura JF, Borges-Pereira J, Costa J. Sobre a possibilidade da ocorrência de doença Chagas autóctone em Roraima, Amazônia brasileira. 2000-2001. Rev Inst Med Trop 2004; 47(1): 45-54.
13. Camargo LMA, Moura MM, Engracia V, Pagotto RC, Basano AS, Pereira da Silva LH et al. A rural community in a Brazilian Western Amazon Region: Some Demographic and Epidemiological Patterns. Mem Inst Oswaldo Cruz 2002; 97: 193-5.

14. Silveira AC. Situação do controle da transmissão vetorial da doença de Chagas nas Américas. Cad Saúde Pública 2000: 16 (S2): 35-42.

15. Fernandes AJ, Diotaiuti L, Dias JCP, Romanha AJ, Chiari E. Inter-relações entre os ciclos de transmissão do Trypanosoma cruzi no município de Bambuí, Minas Gerais, Brasil. Cad Saúde Pública 1994: 10(4): 473-80.

16. Romana CA, Pizarro JC, Rodas E, Guilbert E. ROMAÑA Palm trees as ecologicators of risk areas Chagas disease. Transactions of the Royal Society of Tropical Medicine and Hygiene 1999; 93: 594-5.

17. Gonçalves-Gurgel R, Ramalho ED, Palma ART, Romaña CA, Cuba-Cuba CA. Distribuíção espacial de população de triatomíneos (Hemipteras: Reduviidae) em palmeiras da espécie Mauritia flexuosa no Distrito Federal, Brasil. Rev Soc Bras Med Trop 2004; 37(3): 241-7.

18. Valente VC, Valente SAS, Rodrigues CA, Souza GCR, Noireau F. Lopes CM et al. Estudo preliminar da eficiência de captura de triatomíneos silvestres utilizando armadilhas com fita adesiva em Bragança Pará. Rev Soc Bras Med Trop 2000; (S2): 94-5.

19. Coura JR, Junqueira ACV, Bóia MN, Fernandes O. Chagas Disease: from Bush to Huts and Houses. Is it the case of the Brazilian Amazon? Mem Inst Oswaldo Cruz 1999; 94:(S1): 379-84.

20. Dias JCP, Vinhaes MC, Silveira AC, Schofield CJ, Cardoso B, Coura JR.. Pesquisa prioritárias sobre doença de Chagas na Amazônia: agenda de curto-médio prazo. Rev Soc Bras Med Trop 2001; 34: 61-2.

21. Barreto MP, Albuquerque DRR, Funayama GK. Estudo sobre reservatórios e vectores silvestres do Trypanosoma cruzi. XXXVI: Investigação sobre triatomíneos de palmeiras no município de Uberaba, MG, Brasil. Rev Bras Biol 1969; 29: 577-88.

22. Miles MA, Souza AD, Povoa M. Chagas disease in the Amazônia basin. Ecotopes of tem triatomine bug species (Hemiptera, Reduviidae)from the vicinity of Belém, Pará state, Brazil. J Med Entomol 1991; 18: 266-78.

23. Zeledón R. Some historical facts and recent issues related to the presence of Rhidnius prolixus (Stal,1859) (Hemiptera: Reduviidae) in central America. Entomol Vect 2004; 11(2): 233-46. Disponível em www.ugf.br/ editora. 
24. Rocha DS, Fonseca AH, Costa FA, Jurberg J, Galvão C. Desenvolvimento de Rhodnius pictipes Stal,1872 alimentado através da membrana de silicone e em camundongos (Hemíptera, Reduviidae, Triatominae). Mem Inst Oswaldo Cruz 1997; 92(4): 553-8.
25. Albajar PV, Laredo SV, Terrazas MB, Coura JR. Miocardiopatia dilatada em pacientes com infecção chagásica crônica. Relato de dois casos fatais do Rio Negro, Estado do Amazonas. Rev Soc Bras Med Trop 2003; 36(3): 401-7.

Recebido em: 25/05/06

Versão final reapresentada em:29/10/07

Aprovado em: 19/03/08 